\title{
Fingerprint Recognition Based on Adaptive Neuro-Fuzzy Inference System
}

\author{
Tripti Rani Borah, Kandarpa Kumar Sarma, and Pran Hari Talukdar \\ 1 Department of Computer Science, Gauhati University Guwahati, Assam, India \\ \{tbtriptidas29, kandarpaks\} @gmail.com \\ 2 Dept. of Electronics and Communication Technology, Gauhati University Guwahati, Assam, \\ India \\ 3 Department of Instrumentation and USIC, Gauhati University Guwahati, Assam, India \\ phtalukdar@gauhati.ac.in
}

\begin{abstract}
Fuzzy logic (FL) is a powerful problem solving methodology receiving wide spread acceptance for a range of applications. FL is also considered for image understanding applications such as edge detection, feature extraction, classification and clustering. It provides a simple and easy way to draw a definite conclusion from ambiguous, imprecise or vague information. Like Artificial Neural Network (ANN) models, some fuzzy inference system (FIS)s have the capability of universal approximation. The adaptive neuro-fuzzy inference system (ANFIS) belongs to the class of systems commonly known as neuro-fuzzy systems (NFs). NFs combines the advantages of ANN with those of fuzzy systems. An ANFIS based identification system is described here which uses fingerprint as an input. Experiments are carried out using a number of samples. Obtained results show that the system is reliable enough for considering it as a part of a verification mechanism.
\end{abstract}

\section{Introduction}

To deal with security problems, the use of biometric-based technology (specifically through fingerprint, face, speech etc.) are considered, because such biometric factors have unique characteristics and are related to specific detection and decision states. The related processing technology has reached a high degree of maturity, such as allowing successful application on secure authentication [1]. Fingerprints have been used for over a century and are the most widely used form of biometric identification. The fingerprint of an individual is unique and remains unchanged over a lifetime [2]. Fingerprints are graphical flow-like ridges and valleys present on the surface of human fingers. Typically, there are two prominent types of minutiae (ridge endings and ridge bifurcations) that constitute a fingerprint pattern. A fingerprint can be represented by the minutiae locations, types and attributes like orientation. A good quality fingerprint image typically has about 40 to 100 minutiae, but a dozen of minutiae are considered sufficient to identify a fingerprint pattern. An fingerprint recognition system (FRS) is one of the popular biometric techniques and is an automated method of verifying a match between fingerprint images [3]. Though fingerprint of an individual is unique and doesnot change over a lifetime, for a large number of such samples, usually seen in a biometric authentication 
system, the classifier must have the ability to capture and retain the minute variations observed in the applied patterns. Fuzzy systems are useful for dealing with uncertainty. Fuzzy logic (FL) is a powerful problem solving methodology receiving wide spread acceptance for a range of applications. FL is also considered for image understanding applications such as edge detection, feature extraction, classification and clustering. It provides a simple and easy way to draw a definite conclusion from ambiguous, imprecise or vague information. Like Artificial Neural Network (ANN) models, some fuzzy inference system (FIS)s have the capability of universal approximation. The adaptive neuro-fuzzy inference system (ANFIS) belongs to the class of systems commonly known as neuro-fuzzy systems (NFs). NFs combines the advantages of ANN with those of fuzzy systems. The ANFIS combines the advantages of neuro and fuzzy based systems and proves to be adaptive and robust in dealing with finite variations. Here, we propose a fingerprint recognition system (FRS) based on ANFIS. Experimental results show that the proposed approach is fast, reliable and robust for a range of conditions. An ANFIS can be configured and trained to handle range of variations observed in the texture of the fingerprint. The specialty of the work is associated with the fact that if the ANFIS is configured properly it can tackle the variations in the fingerprint images. This way the approach provides the insights for developing a system which requires these samples for verification and authentication. Some of the related works are [7] to [8].

The rest of the paper is organized as follows. Section[2 provides the proposed system model and implementation details. All experimental results and related discussions are provided in Section 3. This paper is concluded by summing up the work in Section 4.

\section{System Model and Implementation Details}

The ANFIS has a processing system which requires sub-blocks of fuzzification (done using membership functions (MF)), fuzzy rules for inference generation and defuzzification. The ANFIS combines the advantages of ANN with those of fuzzy systems. Here, we consider Sugeno fuzzy model [4]. A generic FRS using ANFIS in block diagram form is shown in Figure 1 The first module is image acquisition which is followed by preprocessing. The feature extraction stage enables the capture of vital information about the input samples. These are next fuzzified using certain MFs. Classification stage is the key component of the system and determines the system's performance to a large extent. ANFIS is used as classifier and it produces the correct result by classifying the feature extracted templates and matching these features with known patterns in the feature database. An ANFIS basic building block diagram is shown in Figure2 with its five layers of different functions which constitute the system. The first layer consists all the adaptive nodes with membership values as outputs which is followed by the next layer consisting of the fixed node which multiplies the incoming signals and gives the product as output. The next layer is the normalization layer. The fourth layer is an adaptive node which is followed by the fifth layer with the overall output of the model.

In this proposed model, a multi stage approach is used. Fingerprint images are captured by a digital scanner. Preprocessing includes resizing images, normalization, segmentation and thinning etc. The next stage is feature extraction. The thinned images are considered for the minutiae feature extraction. The minutiae feature extraction algorithm extracts the minutiae features required for our purpose. Here, Crossing Number 


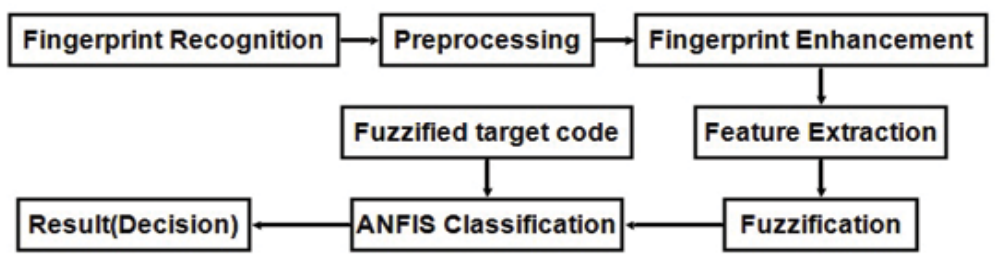

Fig. 1. Process logic of the complete system

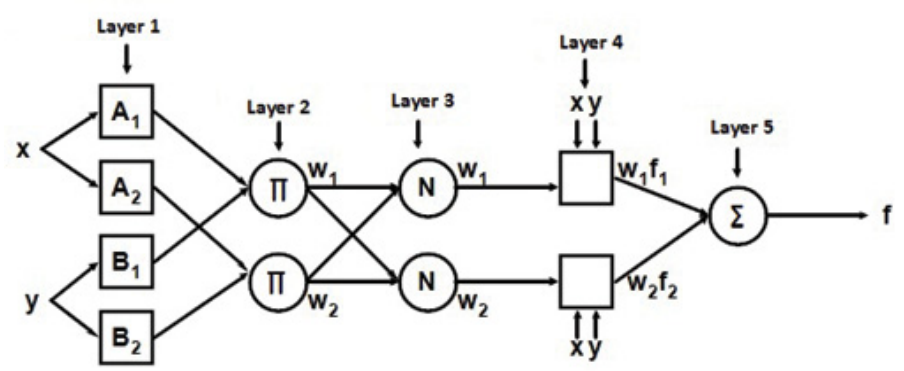

Fig. 2. Building blocks of ANFIS

(CN) method is used for minutiae extraction of fingerprints [5]. The ridge pixel can be divided into bifurcation, ridge ending and non-minutiae point based on it. The $\mathrm{CN}$ algorithm is working on pixel representation 1 or 0 but the decision of minutiae point can be selected for each pixel value. CN method extracts the ridge endings and bifurcations from the skeleton image by examining the local neighborhood of each ridge pixel using a $3 \times 3$ window. The $\mathrm{CN}$ for a ridge pixel $\mathrm{P}$ is given by

$$
C N=0.5 \sum_{i=1}^{8}\left|P_{i}-P_{i+1}\right|, \quad P_{9}=P_{1}
$$

where $P_{i}$ is the pixel value in the neighborhood of $\mathrm{P}$. For a pixel $\mathrm{P}$, its eight neighboring pixels are scanned in an anti-clockwise direction.

After the $\mathrm{CN}$ for a ridge pixel has been computed, the pixel can then be classified according to the property of its $\mathrm{CN}$ value. With this formula, if $\mathrm{CN}=1$ it corresponds to the end point and if $\mathrm{CN}=3$, it corresponds to bifurcation point of minutiae. Other properties of $\mathrm{CN}$ are described in Table 1. While applying this algorithm, border area may be ignored, since there is no need to extract minutiae point on border area of the image that will gives more false minutiae points. After a successful extraction of minutiae, they are stored in a template, which may contain the position, direction, type and quality of the minutiae. Here, ANFIS is used as classifier for recognition. It applies a combination of the least-squares (LS) method and the back propagation gradient descent (BPGD) method for training FIS membership function parameters to emulate a given training data set. Fingerprint with $\mathrm{CN}$ features of length 101 is used for the training of ANFIS for 10 to 60 epochs. The results obtained are average values of atleast ten 
Table 1. Properties of CN

\begin{tabular}{ll}
\hline $\mathrm{CN}$ & Properties \\
\hline 0 & Isolated Point \\
1 & Ending Point \\
2 & Connective Point \\
3 & Bifurcation Point \\
4 & Crossing Point \\
\hline
\end{tabular}

trails for the epochs considered. We have used the generalized bell-shaped MF for doing the fuzzification. This is because the bell-shaped MF provides the most reliable real world to fuzzy conversion as has been known from experiments. We have considered ten such MFs which gives optimum results. Also, we consider the root mean squared error (RMSE) to be the cost functional for training the ANFIS. We consider about forty clean fingerprint samples. From them, a few set of noise corrupted samples are also formed for testing.

Table 2. ANFIS Specifications

\begin{tabular}{ll}
\hline Input Data Size & $\begin{array}{l}\text { Fingerprint with CN features of length 101 } \\
\text { SNR }\end{array}$ \\
ANFIS type & ANFIS with five layers \\
ANFIS training method & $\begin{array}{l}\text { Least-squares method with the back propagation } \\
\text { gradient descent method }\end{array}$ \\
Average training epochs & 10 to 60 \\
Total number of membership functions & 10 \\
Type of membership function & $\begin{array}{l}\text { Generalized bell-shaped built-in membership func- } \\
\text { tion }\end{array}$ \\
RMSE with 3 MFs at 60 epoch & 0.01 \\
RMSE with 10 MFs at 50 epoch & 0.005 \\
\hline
\end{tabular}

Table 3. Average success rates achieved between a few numbers of training epochs with ANFIS and ANN

\begin{tabular}{lllll}
\hline $\begin{array}{l}\text { Observation } \\
\text { No. }\end{array}$ & Epochs by ANFIS & Epochs by ANN & $\begin{array}{l}\text { \%Success rate with } \\
\text { ANFIS Success rate with }\end{array}$ & ANN \\
\hline 1 & 10 & 15 & 88 & 86 \\
2 & 20 & 32 & 92 & 90 \\
3 & 30 & 47 & 94.5 & 92 \\
4 & 40 & 61 & 95.2 & 93 \\
5 & 50 & 76 & 96.5 & 94 \\
6 & 60 & 91 & 98 & 96 \\
\hline
\end{tabular}




\section{Experimental Details and Results}

The performance of FRS is analyzed in terms of computational speed and reliability. The overall computational time taken by the system is reduced to a greater level. The obtained results are compared with [6] and found that the ANFIS generate comparable RMSE values but the computational speed is significantly better. The ANFIS shows at least $50 \%$ increase in computational speed which establishes its usefulness. Further, the RMSE convergence is completed in less number of epochs. Thus the proposed approach is suitable for verification and authorization for the design of FRS. A total of 40 identical fingerprint images have been provided to the system for training, validation and testing of the system. After extensive training, the system is subjected to certain variations with signal to noise ratio (SNR) ranging between 0 to $3 \mathrm{~dB}$ to achieve robustness and proper recognition. The ANFIS considered is configured using the specifications shown in Table 2. The average RMSE convergence plot shown by the ANFIS during training while configuring the FRS is shown in Figure 3. The epochs are between ten to sixty and the success rate is around 88 to $98 \%$. The training time required is between 8 to 10 seconds for a set of ten samples each. The results are derived by performing ten trials for each of the sample sets and the average results are quoted.

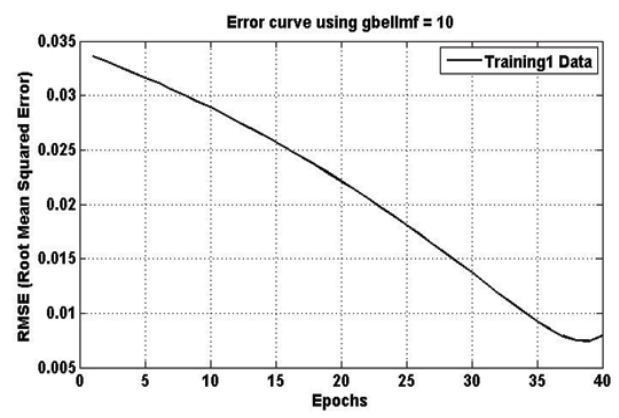

Fig. 3. Average RMSE convergence of the ANFIS upto 40 epochs with 10 MFs

\section{Conclusion}

Here, we described a FRS where the ANFIS forms a critical decision support system. The experimental results show that the proposed approach is at least $50 \%$ more efficient compared to ANN based approaches reported earlier. The strength of the proposed system is its speed, computational efficiency, robustness and high precision which shall make it suitable for certain application. The system proposed here is reliable and efficient enough to be a part of a biometric verification system. The future scope of this work is to enhance the ANFIS architecture to achieve high classification accuracy. The overall performance of the system can be enhanced further by considering more number of samples and variations and by using of better preprocessing techniques with ANFIS based blocks. 


\section{References}

1. Jain, A., Hong, L., Pankanti, S.: Biometric Identification. Communications of the ACM 43(2), 91-98 (2000)

2. Jain, A., Hong, L., Pankanti, S., Bolle, R.: An identity authentication system using fingerprints. Proceedings of the IEEE 85, 1365-1388 (1997)

3. Thai, R.: Fingerprint Image Enhancement and Minutiae Extraction. B. Eng. thesis, The University of Western Australia (2003)

4. Ross, T.J.: Fuzzy logic with Engineering Applications, 2nd edn. Wiley India, New Delhi (2008)

5. Maltoni, D., Maio, D., Jain, A.K., Prabhakar, S.: Handbook of Fingerprint Recognition, 2nd edn. Springer Professional Computing, Berlin (2009)

6. Borah, T.R., Sarma, K.K., Talukdar, P.H.: Fingerprint Recognition using Artificial Neural Network. International Journal of Electronics Signals and Systems (IJESS) 3(1), 98-101 (2013)

7. Jang, R.J.: ANFIS: Adaptive-Network Based Fuzzy Inference System. IEEE Transaction on Systems, Man and Cybernetics 23(3) (1993)

8. Hsieh, C.T., Hu, C.S.: An Application of Fuzzy Logic and Neural network to Fingerprint Recognition. In: Proceedings of IEEE-Eurasip Nonlinear Signal and Image Processing (NSIP 2005), Japan, vol. 20 (2005) 